\title{
Las Tic como herramienta mediadora para el fortalecimiento del valor de la responsabilidad ${ }^{1}$

\author{
Tic as a mediating tool for strengthening \\ the value of responsibility
}

DOI: http://dx.doi.org/10.17981/cultedusoc.9.3.2018.63

Artículo de investigación. Fecha de recepción: 15/06/2018. Fecha de aceptación: 27/11/2018

\author{
Miguel Vargas Yanci; Aníbal Orozco; Eduardo Bocanegra De La Cruz; \\ Ana Cantillo Orozco; Ana Cantillo Orozco; Nohora Pertuz Ospino; \\ Ana Ortega Orozco; Mónica Polo Gamarra; Celmira Montenegro Álvarez; \\ Rafael Morales Cervantes; Martha García Barrios; \\ Martha Castro De La Cruz; Rafael Morales Bocanegra ${ }^{2}$ \\ Institución Educativa Distrital de Carreto municipio del Piñón, Magdalena (Colombia)
}

Para citar este artículo:

Vargas, M., Orozco, A., Bocanegra, E., Cantillo, A., Cantillo, A., Pertuz, A., Ortega, A., Polo, M., Montenegro, C., Morales, R., García, M., Castro, M. y Morales, R. (2018). Las Tic como herramienta mediadora para el fortalecimiento del valor de la responsabilidad. Cultura. Educación y Sociedad 9(3), 529-534. DOI: http://dx.doi.org/10.17981/cultedusoc.9.3.2018.63

\section{Resumen}

El presente artículo muestra los resultados del estudio la tecnología de la información y la comunicación como herramienta mediadora para el fortalecimiento del valor de la responsabilidad. En él se plantea que frente al avance desmesurado de esas tecnologías a nivel mundial, y su uso expandido en cada una de las esferas en que se desenvuelve el ser humano, se hace necesario asumir este reto en los planteles educativos, para además de utilizarlo como medio de aprendizaje, nos permita implementarlo como estrategia para superar dificultades en este proceso. El propósito es impactar positivamente en la interacción de estudiantes y educadores, convirtiéndose en una herramienta de estudio para el adecuado desarrollo de las competencias en los estudiantes, con la intención de utilizarlas transversalmente en la práctica pedagógica como estrategia para fomentar de la responsabilidad en los estudiantes, con el apoyo de los docentes de la institución.

Palabras clave: Tecnología de la información y la comunicación, estrategia pedagógica mediadora, formación de la responsabilidad.

\section{Abstract}

This article shows the results of the Las Tic study as a mediating tool for strengthening the value of responsibility. It states that in the face of the advancement of Information and Communication Technologies (ICT) worldwide, and its expanded use in each of the spheres in which the human being develops, it is necessary to assume this challenge in the educational sites, in addition to using it as a means of learning, allow us to implement it as a strategy to overcome difficulties in this process. The above aims to positively impact the interaction of students and educators, becoming a study tool for the adequate development of competencies in students, in which the fundamental purpose of this research is to use ICT as a pedagogical strategy for the promotion of responsibility in students supported by the teachers of the institution.

Keywords: Information and communication technology, pedagogical mediating strategy, responsibility formation.

1 Este artículo ha sido derivado del Proyecto Fortalecimiento de la Cultura Ciudadana y Democrática en CT+I a través de la IEP apoyada en TIC en el Dpto. del Magdalena

2 Docentes de la institución Educativa Distrital de Carreto en el municipio del Piñón-Magdalena.

- The author; licensee Universidad de la Costa - CUC.

Cultura, Educación y Sociedad vol. 9 no. 3, pp. 529-534. Diciembre, 2018

Barranquilla. ISSN 2389-7724 Online 


\section{Introducción}

Considerando el gran avance de las Tecnologías de la Información y la Comunicación (TIC) en este mundo globalizado, se ha expandido en cada una de las esferas en que se desenvuelve el ser humano, por lo que se hace necesario asumir el reto en los planteles educativos, para utilizarlo como medio de aprendizaje. Su implementación como estrategia para enriquecer el aprendizaje y superar dificultades en este proceso, se dirige a impactar positivamente en la interacción de estudiantes y educadores, convirtiéndose en una herramienta de estudio para el adecuado desarrollo de las competencias en los estudiantes (Comisión Económica para América Latina y el Caribe-CEPAL, 2010; Díaz-Barriga, 2005).

El grupo de investigación "Investigando ando", surge precisamente en los educandos de la Institución Educativa Distrital de Carreto, por la necesidad de potencializar el valor de la responsabilidad como pilar fundamental para su desarrollo personal en el aula de clases. El grupo está conformado por trece docentes de este instituto, a nivel de bachillerato; dos docentes de la sede Escuela Rural de Varones, y un docente de la sede Escuela Rural de Niñas.

$\mathrm{Al}$ incluir la Investigación como Estrategia Pedagógica (IEP) en nuestro quehacer docente, en palabras de Mejía y Manjarréz (2011), quienes reconocen que la inserción de la investigación es una forma de promover cambios en las ideas, en las realidades y en el entorno de los participantes, y con ello, es una manera de situarse crítica y éticamente en el mundo cercano y mediato.

De esta manera se transforman las prácticas pedagógicas (Red Distrital de Docentes Investigadores-REDDI, 2017), y se incorporan nuevas formas de generar conocimiento desde el planteamiento de interrogantes, lo cual conduce a buscar de manera estratégica solución a las pro- blemáticas que se viven al interior de la institución educativa, y que ayudan al estudiante a potencializar sus capacidades no solo en el entorno educativo sino en las diferentes áreas en que se desenvuelva.

uego de la exploración o diagnóstico en cuanto a las temáticas que generan dificultades en cada una de las sedes de la institución, se llegó al consenso de trabajar el proyecto de investigación dirigido a potencializar la responsabilidad por parte de los estudiantes, ya que se observó dificultad en el cumplimiento de sus deberes.

Actualmente se evidencia en la sociedad pérdida de los valores, los cuales regulan el comportamiento y permiten que exista una sana convivencia. Para Arrieta (2016) la educación integral y laica forma ciudadanos para el mundo de hoy. En la Institución Educativa Distrital de Carreto se conserva el interés por lograr el desarrollo de educandos formados integralmente; se busca fortalecer los comportamientos de los estudiantes que asuman sus tareas escolares con responsabilidad.

Sin embargo, debido a que los estudiantes manifiestan bajo rendimiento académico, lo cual puede estar relacionado, entre otros factores, con la falta de responsabilidad con las actividades asignadas, emerge la necesidad de proponer estrategias significativas para transformar esa realidad social.

En el aula de clases, el proceso de enseñanza aprendizaje no se observa muy dinámico, por ello es preciso intensificar el esfuerzo por transformar el ambiente de aprendizaje en pro de lograr mejores resultados en los registros internos.

Se requiere del ejercicio permanente de estrategias didácticas que logren potencializar las actitudes y aptitudes, las cuales son esenciales para favorecer el campo de aprendizaje, aquí el docente adquiere una gran responsabilidad para mantener y mejorar la motivación del estudiante (Sancho, s/f.) 
Como herramienta educativa, la implementación de las TIC en las instituciones de educación ha sido paulatina; no se observa aun el énfasis que el maestro debe tener como un facilitador y el estudiante como constructor de su propio conocimiento o aprendizaje. En el enfoque constructivista se promueven las actividades basadas en experiencias ricas, dinámicas y pertinentes al contexto (Hernández, 2008). Desde la perspectiva de autores como Marín Niebles, Sarmiento y Valbuena (2017), la integración de las TIC al proceso educativo resulta clave, por cuanto actúan como herramientas o entornos de aprendizaje en la mediación didáctica para el desarrollo de competencias.

El constructivismo ofrece un nuevo paradigma para esta nueva era de información motivado por las nuevas tecnologías que han surgido en los últimos años. Con la llegada de estas tecnologías (wikis, redes sociales, blogs...), los estudiantes no sólo tienen a su alcance el acceso a un mundo de información ilimitada de manera instantánea, sino que también se les ofrece la posibilidad de controlar ellos mismos la dirección de su propio aprendizaje (Hernández, 2008; Carretero, 1999)

Sin embargo, para que este crecimiento y enriquecimiento de los procesos de enseñanza aprendizaje pueda darse, es necesario que los docentes tengan capacidad, conocimientos y habilidades para el manejo de tecnologías educativas en el aula, y administración de plataformas (Suárez, 2005), lo cual sería de gran utilidad para la disminución de la monotonía de las clases magistrales en las jornadas escolares y por ende conllevaría al incremento del interés por parte de los estudiantes.

\section{Metodología}

Dentro de la investigación se plantearon los siguientes parámetros metodológicos. En el diseño se utilizó el modelo de la inves- tigación cualitativa, el cual consiste en una categoría de diseños de investigación que extraen descripciones a partir de observaciones que adoptan la forma de entrevistas, narraciones, notas de campo, grabaciones, transcripciones de audio, vídeo, registros escritos de todo tipo, fotografías o películas y artefactos (Pérez, 2012; Hernández 2014).

El modelo investigativo trabajado es la investigación acción, que es una metodología de investigación orientada a la práctica educativa cuyo propósito general es mejorar las prácticas educativas. Sus características principales son la transformación y mejora de una realidad educativa (Cerda, 1997).

La unidad de análisis empleada estuvo conformada por un total de 45 estudiantes y 13 maestros de bachillerato la Institución Educativa Distrital de Carreto.

Los instrumentos de recolección de información utilizados fueron: la bitácora $o$ diario de campo, relatorías individuales, institucionales, observación directa, encuestas a los estudiantes y docentes de la institución (Tamayo y Silva, 2008)

En cuanto al procedimiento para recoger los datos, se utilizaron relatorías individuales, institucionales, diario de campo y la aplicación de una encuesta a los estudiantes y docentes de la institución. Se identificó que algunos docentes trabajan metodologías para apoyar a los estudiantes en el proceso de enseñanza aprendizaje, sin embargo, para los estudiantes esto no ha sido suficiente puesto que siguen desmotivados y teniendo problemas por la falta de responsabilidad a la hora de entregar sus informes y cumplir con sus compromisos.

\section{Resultados}

Se logró evidenciar de acuerdo a los resultados de la escala aplicada, que el $86 \%$ de los estudiantes presentan gran interés en que los docentes utilicen las TIC como herramientas para el desarrollo de las clases. 
En las encuestas a los docentes, se pudo apreciar que algunos docentes trabajan metodologías para apoyar a los estudiantes en el proceso de enseñanza aprendizaje, sin embargo, para los estudiantes esto no ha sido suficiente puesto que siguen desmotivados y teniendo problemas por la falta de responsabilidad a la hora de entregar sus informes y cumplir con sus compromisos.

Los docentes están en el proceso de reconocimiento de nuevas metodologías a emplear en el aula de clases, tienen la mente abierta al cambio y con disposición de lograr cambios significativos en la educación de los estudiantes.

Así mismo, después de analizar los resultados obtenidos en la presente investigación y a la revisión de la literatura, se considera oportuno emplear estrategias para fomentar la responsabilidad en nuestros estudiantes y motivar a los docentes a transformar sus prácticas pedagógicas; estrategias tales como: aprendizaje basado en problemas, estudio de caso, aprendizaje basado en proyectos y aprendizaje cooperativo.

\section{Conclusiones}

El proyecto investigativo desarrollado permite concluir que cada aspecto que intervenga en la vida de los estudiantes será un factor predisponente en su desempeño en cada aspecto de su vida. En este proyecto se utilizaron las TIC como estrategia pedagógica para fomentar el valor de la responsabilidad de los estudiantes, con respecto a sus deberes académicos, lo cual permitió conocer lo que los estudiantes percibían en relación a las estrategias pedagógicas de los docentes.

En muchos casos, estudiantes no cumplían con sus deberes a raíz de que de dichas estrategias no eran lo suficientemente motivantes para ello. Con lo anteriormente expuesto podemos resaltar la importancia de esta investigación, pues evidenció la necesidad de realizar cambios curriculares y sobretodo estratégicos al momento de planear e impartir las clases.

El uso de las TIC permitirá motivar a los estudiantes para mejorar su desempeño académico y sentido de responsabilidad con su institución y con ellos mismos. Para los cambios curriculares antes mencionados, es necesario saber a qué hace referencia y la importancia de la TIC en el ámbito educativo.

Las Tecnologías de la Información y la Comunicación (TIC) son actor fundamental y parte de la cultura académica. Como es de esperar al interior de las instituciones educativas de nivel superior, el recurso tecnológico disponible suele ser cada vez más cuantioso, aunque no lo suficiente frente a lo que se espera; de hecho, es importante considerar que la presencia de tecnologías no es sinónimo del uso adecuado de las mismas. (Karam, Buitrago, Fagua y Romero, 2013)

Tendiendo claridad sobre lo que se planeta de la TIC, es pertinente hablar sobre cómo se ha evaluado este aspecto en la educación, y es necesario seguir investigándolo. Según afirma Hederich, (1999):

La medición del impacto de TIC en la educación, normalmente ha sido evaluada a partir del número de computadores en las instituciones y el número de docentes que han sido capacitados en el tema. Es necesario definir indicadores claros y suficientes del impacto en la mejora de los programas o en las innovaciones realizadas en el proceso educativo. Es necesario investigar los aprendizajes que generan las TIC en los aspectos motivacionales y relacionarlos también con los estilos de aprendizaje (1999).

A lo largo del tiempo, han ocurrido cambios importantes, según Karam et al. (2013), los organismos multilaterales y centros de investigación de diferentes lugares del mundo, Han tendido a implementar modelos que definen estándares e indicadores, en aspectos fundamentales para la búsqueda de una correcta incorporación pedagógica que permita trascender del tema de 
infraestructura, de hardware, software y telecomunicaciones instalados en las instituciones. La incorporación de las TIC en las instituciones educativas ha pasado por varias etapas: 1) Sistematización de los sistemas administrativos, 2) dotación de aulas de informática, 3) sistematización de los sistemas académicos, 4) uso de TIC en clases presenciales diferentes a informática y, 5) uso de aulas virtuales y espacios WEB.

En cuestiones de resultados, la capacidad de difusión de la información a través de la TIC, puede facilitar muchos proyectos en el aula, ya que el acceso a esa información es más puntual al momento de desarrollar la práctica pedagógica. Las etapas a lo largo del tiempo han ido cambiando, según la necesidad y el valor que se le han dado a la misma en las instituciones educativas.

Tal como plantean los autores Betancur, Carmona, Contreras, Karam, Maestre, Romero y Uribe (2014): Ciertas estrategias con las herramientas de las TIC que han favorecido al aprendizaje, la observamos en la introducción de la franquicia gaming. Entendiendo por la Gamificación, el uso de mecánicas de juego en otro tipo de ámbitos, con el objetivo de atraer y retener audiencias; minimizar los tiempos de adopción de productos o servicios; animar a la gente a realizar tareas que consideran tediosas o difíciles y fortalecer procesos de aprendizaje.

En esta fusión de las TIC con los juegos, específicamente con el videojuego, se han desarrollado formas muy entretenidas e interactivas de desarrollo de habilidades y destrezas que pueden resultar muy atractivas para el alumno. El proceso educativo tiene el desafío de trabajar mucho para que las experiencias que se propongan en las instituciones educativas resulten tanto o más atractivas, y si no, por lo menos utilizar esas oportunidades para atraer al alumno a responsabilizarse de la búsqueda de su desarrollo y la conciencia de cuáles son los riesgos y consecuencias de dedicar el tiempo a actividades lúdicas, descuidando sus experiencias de aprendizaje.

En conclusión, las TIC desde cualquier ámbito fortalecen todo tipo de proceso educativo, simplemente deben llevarse al campo educativo de la mejor manera y con las mejores adaptaciones curriculares o en proyectos transversales para el fortalecimiento de la personalidad del alumno. Las instituciones educativas deben plantearse caminos para aprovechar las herramientas de las TIC discutiendo y reflexionando con sus alumnos y con la comunidad en general sobre los riesgos y las desventajas que pueden presentarse, atender cómo se manifiestan esas desventajas en su comunidad educativa y las posibilidades de contextualizar los recursos disponibles, con la intención de aprovechar en cuanto sea posible la utilización de las redes y las TIC para motivar a los alumnos a la investigación sobre temáticas curriculares, o las que puedan interesarles a los actores del proceso formativo; de tal manera que apunten a fortalecer rasgos personales y ciudadanos, especialmente a potenciar la responsabilidad en el aprendizaje.

\section{Referencias}

Arrieta, M. (2016). Los masones en el mundo: Geopolítica masónica. La historia de los hijos de la Luz. Córdoba: Editorial Almuzara.

Betancur, S.; Carmona, L.; Contreras, R.; Karam, J., Maestre, N.; Romero, Y. y Uribe, S. (2014). Videojuegos y TIC como estrategias pedagógicas: Formación para el uso seguro de internet. Cultura, Educación y Sociedad, 5(1), 91-107.

Carretero, M. (1999). Constructivismo y educación. México, D.F.: Progreso.

CEPAL. (2010). Avances en el acceso y el uso de las Tecnologías de la Información y la Comunicación en América Latina y el Caribe 2008 - 2010. Chile: ONU. 
Cerda, H. (1997). La investigación total. Bogotá, D.C.: Magisterio.

Díaz-Barriga, F. (2005). Enseñanza situada. Vínculo entre la escuela y la vida. México, D.C.: Mc Graw Hill. Recuperado de https://www.uv.mx/rmipe/.../Ensenanza-situada-vinculo-entre-la-escuelay-la-vida.pdf

Hernández, S. (2008). El modelo constructivista con las nuevas tecnologías: aplicadoenelprocesodeaprendizaje-Educrea. Educrea. Recuperado de https://educrea. cl/el-modelo-constructivista-con-lasnuevas-tecnologias-aplicado-en-el-proceso-de-aprendizaje/

Hernández, R., Fernández, C. y Baptista, P.(2014). Metodología delainvestigación. México, D.F.: McGraw-Hill Education.

Karam, J., Buitrago, A., Fagua, A. y Romero, Y. (2013). Estrategias de gamificación aplicadas al diagnóstico de la incorporación pedagógica de las TIC en una comunidad académica. Cultura, Educación y Sociedad, 4(1), 55-74.

Marín, F., Niebles, M., Sarmiento, M. y Valbuena, S. (2017). Mediación de las tecnologías de la información en la comprensión lectora para la resolución de problemas aritméticos de enunciado verbal. Espacios, 38(20) Recuperado de http://www.revistaespacios.com/ a17v38n20/a17v38n20p20.pdf
Mejía, M. y Manjarrés, M. (2011). La investigación como estrategia pedagógica una apuesta por construir pedagogías críticas en el siglo XXI. Praxis \& Saber, 2(4), 127. $\mathrm{h}$ t $\mathrm{t} p \mathrm{p}: / \mathrm{d}$ d $\mathrm{x}$. $\mathrm{d}$ o $\mathrm{i}$. org/10.19053/22160159.1127

Pérez, G. (2002). Investigación cualitativa. Retos e interrogantes. II técnicas y análisis de datos. Madrid: La Muralla.

REDDI. (Octubre 14, 2017). Aprendizaje y Didáctica: posibilidades de transformación en la práctica pedagógica. Compartir palabra maestra. Recuperado de https://compartirpalabramaestra.org/ blog/aprendizaje-y-didactica-posibilidades-de-transformacion-en-la-practicapedagogica

Sancho, J. (s/f). Técnicas de enseñanza para mejorar la motivación de los estudiantes. Recuperado de https://educrea. cl/tecnicas-de-ensenanza-para-mejorarla-motivacion-de-los-estudiantes/

Suárez, R. (2005). La Educación. Teorías Educativas. Estrategias de Enseñanza Aprendizaje. Madrid: Trillas

Tamayo, C. y Silva, I. (2008). Técnicas e instrumentos de recolección de datos. Universidad Católica, Los Ángeles de Chimbote. Recuperado de http://www. postgradoune.edu.pe/pdf/documentosacademicos/ciencias-de-la-educacion/23. pdf 